\title{
A utilização de objetos de aprendizagem no estudo do orvalho e da geada no $6^{\circ}$ ano do ensino fundamental
}

\author{
The usage of learning resources in the study of dew and hoarfrost in the 6th grade of elementary \\ school
}

\author{
Mariana Xavier Oliveira, Eliege Cassiele Buffon, Mauro Kumpfer Werlang
}

Departamento de Geociências, Universidade Federal de Santa Maria, Santa Maria, Brasil

\section{Resumo}

A ocorrência do orvalho e da geada é uma situação, conforme o ritmo climático, comum no sul do Brasil. A maioria dos livros didáticos aborda este assunto de forma estática. Isso dificulta que o aluno visualize a dinâmica do fenômeno. Diante dessa dificuldade propõem-se recursos didático-pedagógicos que auxiliem o professor nesta prática e proporcionem ao aluno uma interação com os fenômenos. Diante da possibilidade do aluno interagir com as novas mídias voltadas à educação, os objetos de aprendizagem podem servir como elo entre essas mídias e o ensino da climatologia, mais especificamente, no ensino do orvalho e da geada. Com o auxílio de um aparelho decodificador de vídeos digitais (DVD) é possível tornar as aulas mais dinâmicas e interativas, pois através deste instrumento e da criatividade do professor, é possível tornar as aulas, e especialmente as de geografia, mais atrativas. Dentro dessa perspectiva que foi proposto o presente trabalho, o qual se concretizou numa escola da periferia de Santa Maria, RS. Utilizando períodos de aulas do quinto ano essas se tornaram repletas de cooperativismo entre os acadêmicos. A avaliação dos resultados mostrou-se positivo, o que demonstra a eficácia do objeto de aprendizagem aplicado.

Palavras-chave:: recursos didático-pedagógicos; objeto virtual de aprendizagem; fenômenos climáticos; orvalho e geada.

\section{Abstract}

The occurrence of dew and frost is a situation, accordint to the climatic rhythm, common in the southern Brazil. Most textbooks discusses this subject in a static way. This hinders that the student visualize the dynamics of the phenomenon. Given this difficulty we propose didactic teaching resources to assist the teacher in this practice and provide to the student an interation with the phenomena. Facing the possibility of the student to interact with new media related to education, the learning objects may serve as a link between these media and the teaching of climatology, specificall in the teaching of dew and frost. With the assistance of a digital video decoder (DVD) it was possible to make the lessons more dynamic and interactive, because with this instrument and the creativity of the teacher, it is possible to make the lessons, and specially geography, more attractive. Within this perspective that the present work was proposed, wich materialized in a school on the outskirts of Santa Maria, RS. Using class periods of the fifth year, these became full of cooperativeness among academics. The evaluation of the results was positive, wich shows the effectiveness of the learning object applied.Abstract here Abstract here Abstract here Abstract here Abstract here Abstract here

Keywords: didactic-pedagogical resources; virtual learning resource; climate phenomena; dew and hoarfrost. 


\section{Introdução}

$\mathrm{O}$

mundo passa por constantes transformações, onde as informações são processadas de maneira cada vez mais rápidas e o ensino encontra-se inserido neste processo globalizado. A educação encontra no computador um instrumento auxiliar para o processo de aprendizagem, propondo ao educando novas tecnologias informacionais.

De acordo com Bolfe; Fantini; Costa (2007) a importância da informática e da inclusão digital nas escolas públicas das redes estaduais e municipais de ensino, advém do fato da primeira se constituir numa ferramenta auxiliar ao professor e a segunda em uma medida política para a inserção e promoção social do estudante enquanto cidadão.

É importante que o professor se utilize das tecnologias para que assim a educação e, em particular a geografia, possa se valer dessas novas tecnologias. Nesse contexto, é possível melhorar a interdisciplinaridade abrindo espaços para que a disciplina de geografia melhore a relação com as demais áreas do conhecimento. Considera-se que é imperativa a inter-relação entre os saberes. Nesse sentido Mercado (2002, p.11) observa que "as novas tecnologias e o aumento exponencial das informações levam a uma nova organização de trabalho, em que se faz necessário: a imprescindível especialização dos saberes; a colaboração transdisciplinar e interdisciplinar, o fácil acesso a informação e a consideração do conhecimento como um valor precioso, de utilidade na vida econômica".

Sabe-se que o estudo do clima não se restringe apenas ao estudo de massas de ar e previsões do tempo. Ele é fator determinante de relações sociais e econômicas, condicionando muitas vezes, o grau de desenvolvimento em determinadas regiões do planeta. Assim, é necessário que os alunos tenham conhecimento dos fenômenos locais, como e porque eles ocorrem. Isso é essencial para que eles possam entender as diferenças climáticas que condicionam o ambiente e o seu cotidiano, além de muitos dos seus hábitos e costumes durantes as estações do ano.
O estudo do clima no ensino fundamental necessita ser mais atrativo e dinâmico para que a compreensão por parte dos alunos seja mais efetiva e seu aprendizado mais eficaz. Assim, surgem como alternativa os objetos virtuais de aprendizagem, tanto como ferramenta de auxilio ao professor como instrumento de aprendizagem para os alunos. Deixa de ser apenas um recurso didático adicional e passa a ser um instrumento para dinamizar conteúdos.

Em muitas situações livro didático é o instrumento que, tanto professor quanto aluno dispõem. Nesse sentido e diante dessa realidade, o trabalho propôs o desenvolvimento e a utilização de um objeto virtual de aprendizagem para auxiliar na explicação sobre a formação de fenômenos climáticos do orvalho e da geada.

\section{Objetivos}

O ensino da geografia a partir de livros didáticos, principalmente quanto aos conteúdos referentes a clima nas séries do ensino fundamental, carece de maior detalhamento e/ou aprofundamento. Constata-se, em muitos casos que os conteúdos de climatologia nas séries do Ensino Fundamental, são escassos e reduzidos. Sendo assim ao tratar esses conteúdos no ensino de geografia, principalmente os conteúdos relativos ao clima, necessita-se de recursos didáticos que tornem as aulas mais motivadoras indicando para uma melhor aprendizagem.

Nos últimos anos a informática vem possibilitando aos educadores a utilização de objetos e recursos didático-pedagógicos que os auxiliem no processo de ensino-aprendizagem. Nesse sentido surgem os objetos de aprendizagem como um recurso didáticopedagógico que vêm à complementar esta necessidade. Assim a inserção dos alunos frente às novas tecnologias vem a auxiliar o professor dentro da sala de aula e trazem também como objetivo a inclusão do aluno no meio digital.

Diante da importância da informática aplicada à educação e do desenvolvimento de objetos virtuais de aprendizagem, o trabalho teve como objetivo geral contribuir para a inserção de 
objetos de aprendizagem como prática lúdica e, ainda colaborar com a prática docente. Ainda, com o intuito de auxiliar o professor no ensino geografia face a conteúdos de climatologia, este trabalho teve como objetivo propor uma alternativa didático-pedagógica para explicar a formação dos fenômenos climáticos orvalho e geada.

Partiu-se de um pressuposto de que muitas vezes o professor, mesmo dispondo de bons recursos didáticos pedagógicos, ainda carece de utilização de novas tecnologias da informática disponíveis ao ensino, ou simplesmente apresenta dificuldade para trabalhar e desenvolver objetos virtuais de aprendizagem. Nesse sentido, Konrad (2003) comenta que para desempenhar bons resultados, precisa-se de professores preparados na utilização adequada dos programas e equipamentos. Diante disso, destaca Konrad (2003) que a formação e a capacitação de professores implicam no redimensionamento do papel que o professor deverá desempenhar na formação de seus alunos. A presença de um professor capacitado direciona a formação do educando de acordo com o conteúdo estudado, impedindo possivelmente desvios e distrações com o computador. Precisa-se, portanto, investimento na formação dos professores, sendo necessário que assuma novos papéis pertinentes às mudanças tecnológicas.

Assim o processo de capacitação dos professores para o desenvolvimento de aulas que possam utilizar recursos didático-pedagógicos baseados em novas tecnologias depende de uma formação mais completa, eficaz e direcionada à este processo. Pode-se inferir, então, que o uso destas tecnologias promove a construção do conhecimento e auxiliam o aluno para uma melhor aprendizagem na proposta de uma visualização virtual de fenômenos climáticos.

Nesse sentido, os objetos de aprendizagem podem se constituir em ferramentas dinâmicas, interativas e lúdicas para auxiliar a prática docente e tornar o processo de ensino e aprendizagem mais prazeroso. Também, através da capacidade de animação e simulação, os objetos de aprendizagem podem tornar mais simples a compreensão de fenômenos a serem estudados.

\section{Objetos de aprendizagem}

A Geografia estuda a construção do espaço pelo homem, a partir da forma que eles estão organizados na sociedade e das condições naturais desses espaços. Essas condições são resultantes de diversos fatores, como clima, vegetação, relevo, entre outros, que são apresentados aos alunos muitas vezes sem que eles assimilem esses conteúdos. Nesse sentido, os instrumentos tecnológicos podem ser aliados importantes, pois ampliam as possibilidades da busca do conhecimento e, torna ao mesmo tempo, essa busca pelo conhecimento mais atrativa e dinâmica. Contribuem assim para a capacidade criativa dos estudantes que se fazem construtores dos saberes, auxiliando-os na aprendizagem.

Diante da importância da informática aplicada à educação, Niskier (1985, p.80) destaca que "os professores se vêem às voltas com as necessidades de transmitir uma quantidade de conhecimento em constante progressão. Eles envelhecem rapidamente, até mesmo em função do desenvolvimento científico-tecnológico, obrigando a uma permanente atualização".

A educação deve se colocar diante das inovações propondo um desenvolvimento tecnológico em que o conhecimento está em contínua construção, sendo os novos recursos como o computador, o DVD, e até mesmo os mapas e maquetes, ferramentas que podem contribuir muito para o ensino-aprendizagem dos alunos.

Diante de novos recursos tecnológicos para a aprendizagem, em que o conhecimento pré-fixado cede lugar às contínuas inovações, o livro didático funciona conjugadamente, articula-se muito bem como essas novas tecnologias. Nesse sentido, a utilização de objetos virtuais de aprendizagem vem auxiliar e a ampliar o conhecimento nele expresso. De outro lado, quando o objeto de aprendizagem também não contempla de forma correta todo o conteúdo, o livro entra como subsídio de informação correta, logo, se tem uma relação integradora e complementar entre a utilização do livro didático e objetos de aprendizagem.

Existem várias definições para o conceito de objeto de aprendizagem. Para Tarouco; Fabre; Tamusiunas (2003) objeto de aprendizagem é qualquer recurso suplementar ao processo de 
aprendizagem, que pode ser usado para apoiá-lo $\mathrm{e}$, geralmente, aplica-se a materiais educacionais projetados e construídos, com a finalidade de maximizar as situações de aprendizagem onde o objeto possa ser aplicado.

Uma das definições mais específicas, baseada em Sosteric; Hesemeier (2002) concebe como objetos de aprendizagem desde imagens e gráficos, vídeos, sons e ferramentas, até qualquer outro recurso educacional digital a ser utilizado para fins educacionais e que contenha sugestões sobre o contexto de sua utilização.

Pelas muitas necessidades enfrentadas, tanto por parte dos alunos como dos professores, os recursos da informática e principalmente a utilização de objetos de aprendizagem, se constituem em ferramentas educacionais inovadoras e de extrema importância, pois auxiliam de maneira eficaz os professores no processo ensino-aprendizagem tornando a aprendizagem dos alunos mais produtiva e eficiente.

\section{Metodologia}

A partir do conteúdo, relativo a orvalho e geada, presente no livro didático foi elaborado oobjeto de aprendizagem. Para sua elaboração foram utilizadas as ferramentas do programa MacromediaFlash $®$ e também o aplicativo Corel Draw 12 para a elaboração das figuras e os desenhos da formação do orvalho e da geada. No aplicativo Corel Draw 12, foram desenhados animais, vegetação, montanhas e gotas da chuva. A animação desses desenhos foi realizada no programa MacromediaFlash ${ }^{\circledR}$.

A utilização do objeto de aprendizagem se deu na sala de aula do $6^{-}$ano na Escola Municipal de Ensino Fundamental Professora Maria de Lourdes Bandeira Medina. A figura 1 localiza a Escola em relação à área urbana do município de Santa Maria, RS. O objeto de aprendizagem foi empregado como um recurso complementar ao estudo da formação dos fenômenos do orvalho e da geada, conteúdos que estão contemplados no livro didático adotado na Escola. Por meio do objeto de aprendizagem os alunos puderam observar como se dá a formação desses fenômenos, complementando a proposta do livro didático, uma vez que apresenta figuras que se movimentam, dando ao aluno a possibilidade de visualizar a dinâmica na formação dos fenômenos. No desenvolvimento do objeto de aprendizagem foram propostos questionamentos, como por exemplo: quais os estados da água; porque a água na geladeira se torna gelo; porque a água ferve; por quais razões um cubo de gelo derrete dentro de um copo de água ou refrigerante e, por fim, indagaram-se quais razões que fazem com que se forme o orvalho ou a geada.

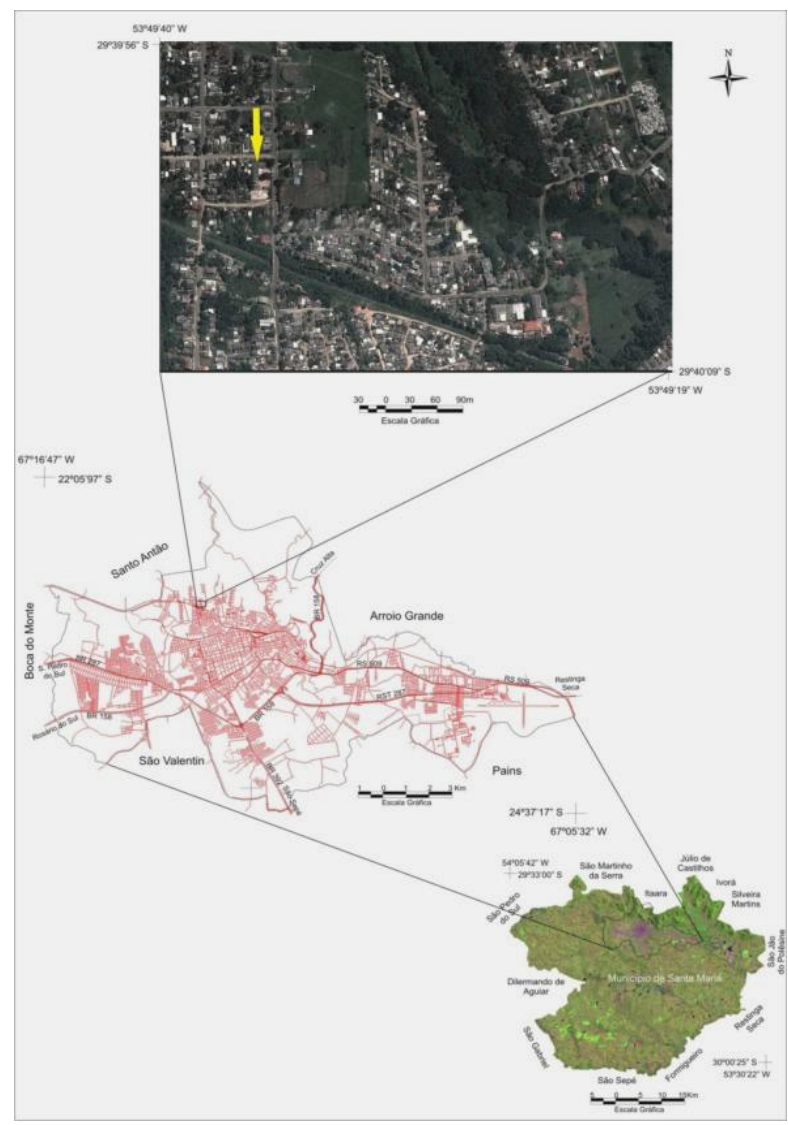

Figura 1: Localização da Escola Municipal de Ensino Fundamental Professora Maria de Lourdes Bandeira Medina em relação à área urbana de Santa Maria, RS.

O trabalho foi desenvolvido em etapas senda que a primeira consistiu em duas aulas teóricas sobre os fenômenos do orvalho e da geada. As aulas foram ministradas pelos pesquisadores e supervisionadas pela professora da disciplina de Geografia da Escola. Uma segunda etapa consistiu na aplicação de um questionário que teve como objetivo avaliar o conhecimento dos alunos em relação aos dois assuntos abordados. Numa etapa seguinte, foi aplicado o objeto de aprendizagem. Após estas etapas, foi reaplicado outro questionário para 
avaliar a evolução da aprendizagem dos alunos em relação aos conhecimentos adquiridos com a aplicação do objeto de aprendizagem. Ainda buscou-se avaliar como os alunos visualizaram a inserção do objeto de aprendizagem a partir de seu envolvimento na atividade. Nesse sentido, procurou-se avaliar os alunos quantitativamente e qualitativamente.

\section{Resultados e Discussão}

Após a realização das etapas do trabalho os alunos puderam interagir com o objeto proposto. Nesse sentido foi observada a orientação de tornar conhecido alguns dos recursos da informática antes de ser apresentado o objeto de aprendizagem. Para isso, as atividades teóricas foram realizadas em duas etapas: uma no inverno (mês de agosto) e outra na primavera (mês de setembro). Após essas etapas teóricas, porém antes de iniciar as atividades de aplicação do objeto de aprendizagem, foi solicitado que os alunos respondessem a um questionário, para que ao final, fosse possível uma avaliação da aprendizagem.

Foram abordados conhecimentos relativos às transformações físicas da água, processo fundamental para o entendimento dos fenômenos orvalho e geada. A figura 2 ilustra um dos momentos dessa etapa das atividades. Nessa atividade, foi demonstrada que essas transformações são comuns e podem ser facilmente percebidas a noite e no amanhecer. Desta forma permitiu-se a exposição das experiências vividas pelos alunos e o reconhecimento dos fenômenos do orvalho e geada bem como porque e como ocorrem. Ainda, os alunos puderam acompanhar a exposição de material ilustrativo e informativo.

Também foram contextualizadas explicações acerca do processo de condensação e solidificação, processos que explicam a formação, respectivamente, do orvalho e da geada ao anoitecer e amanhecer. Ainda, foram contextualizadas explicações acerca dos processos de fusão e vaporização, que explicam o processo de desaparecimento, respectivamente, da geada e do orvalho ao amanhecer. Nesse sentido, buscou-se compreender as relações da transformação dos estados da água com esses fenômenos e, caracterizá-los geograficamente com o inverno e a primavera no sul do Brasil. As figuras 3, 4 e 5 ilustram momentos onde os alunos colaboram e interagem com a atividade proposta.

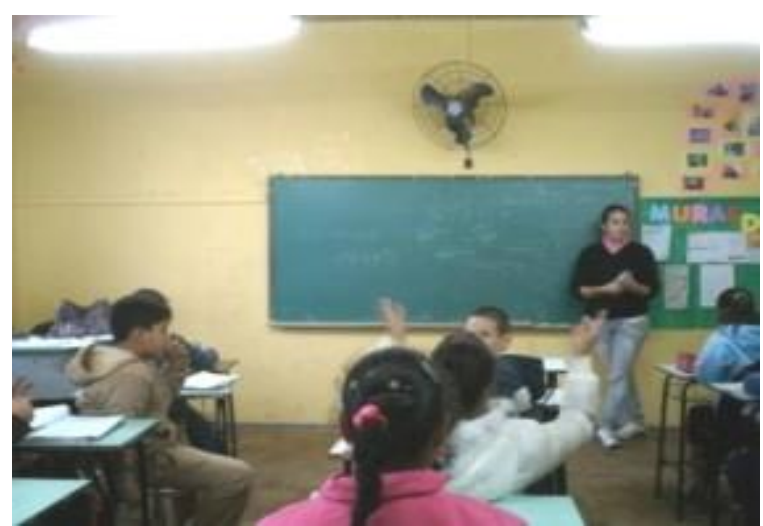

Figura 2: Registro de um momento da exposição para explicação dos conceitos fundamentais para o entendimento do fenômeno do orvalho e da geada.

Fonte: dos autores.

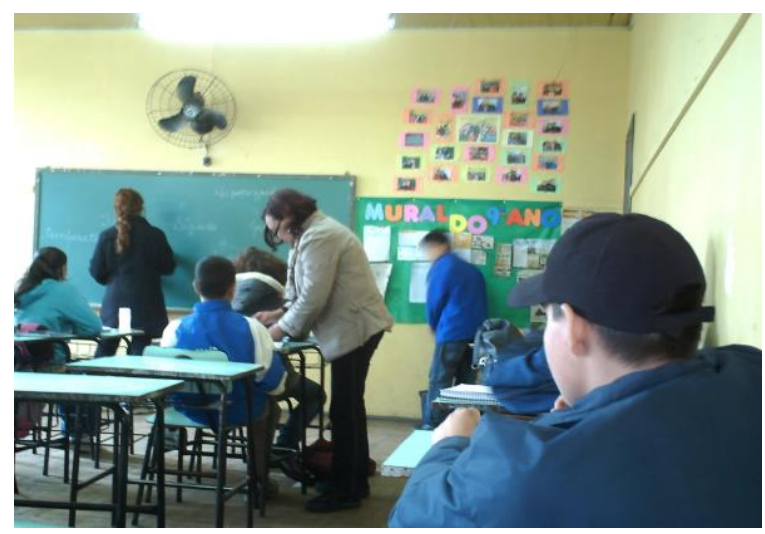

Figura 3: Registro de um momento onde foram contextualizadas situações acerca dos estados e da transformação água e o processo de formação do orvalho e da geada.

Fonte: dos autores.

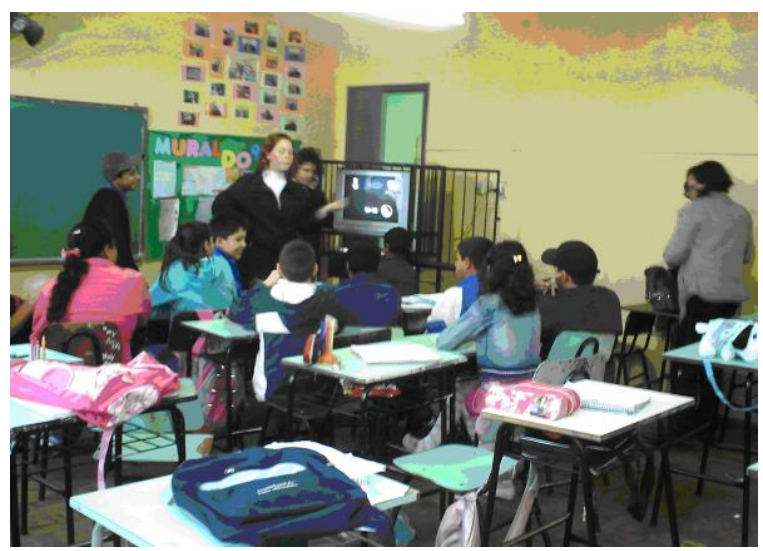

Figura 4: Registro do momento onde a atividade com o objeto de aprendizagem foi proposta.

Fonte: dos autores. 


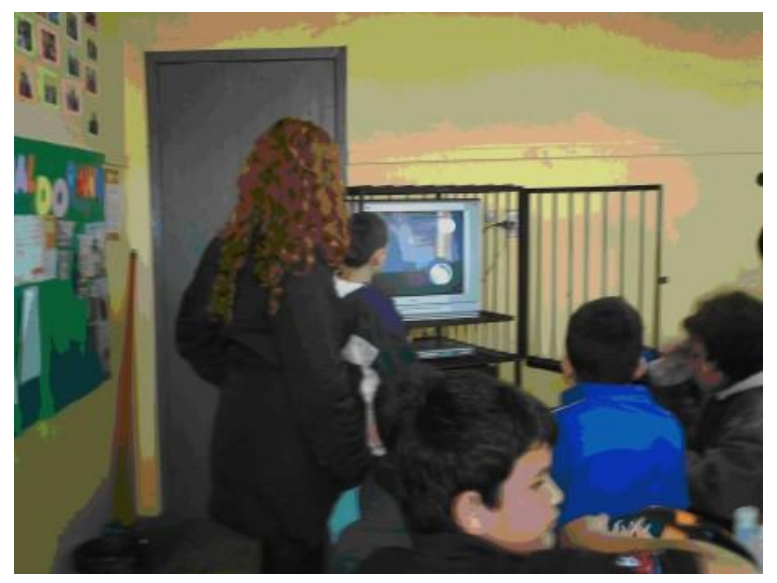

Figura 5: Registro de um momento com o objeto de aprendizagem proposto onde os alunos colaboraram e interagem com a atividade. Fonte: dos autores.

A partir da interação dos alunos com o objeto de aprendizagem foi possível visualizar a dinâmica de formação do orvalho e da geada num período de 24 horas. A partir da observação de uma bússola e com o movimento aparente do sol durante um dia, foi possível perceber o comportamento dos fenômenos desde $o$ nascimento ao ocaso do Sol. Também foram introduzidos no objeto de aprendizagem outros elementos como um termômetro, para a verificação da variação da temperatura e, uma lupa para ampliar uma gotícula de água. $\mathrm{O}$ objeto de aprendizagem proposto reproduziu o período de um dia, por essa razão foi também colocado um relógio para que os alunos pudessem verificar quando, em relação a hora do dia, os fenômenos podem ocorrer.

A figura 6 ilustra, de forma geral, a tela que reproduz o processo de formação do orvalho, e foi capturada do objeto de aprendizagem no momento quando ilustra o processo de deposição da gotícula de água. Nela se pode perceber a mudança que ocorrem na temperatura no decorrer do dia. A figura 7 ilustra uma tela do objeto de aprendizagem no momento da demonstração do processo de formação da geada. Nela se pode observar com destaque à gotícula que está em processo de solidificação formando a geada. Também se pode observar, além das condições de temperatura que propiciam a ocorrência do fenômeno, o horário que ele é mais comum.

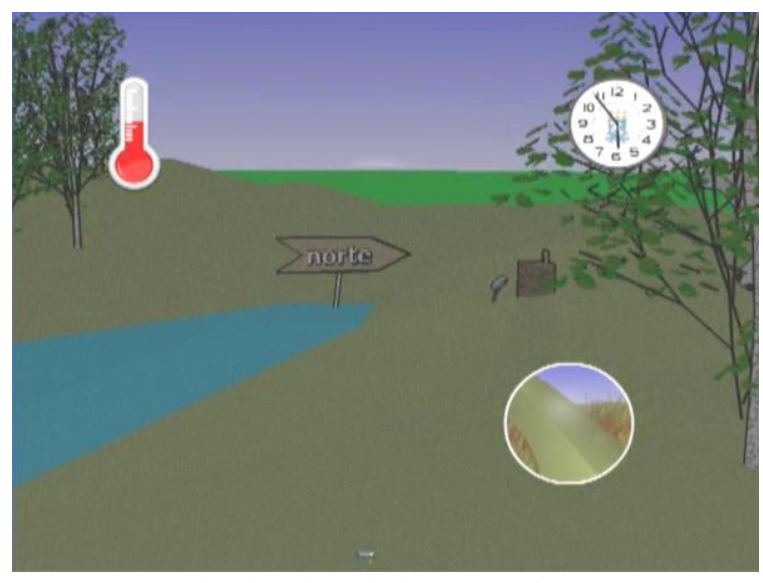

Figura 6: Tela do objeto de aprendizagem capturada do objeto de aprendizagem no momento quando ilustra a deposição de gotícula de água num processo de formação do orvalho Fonte: dos autores.

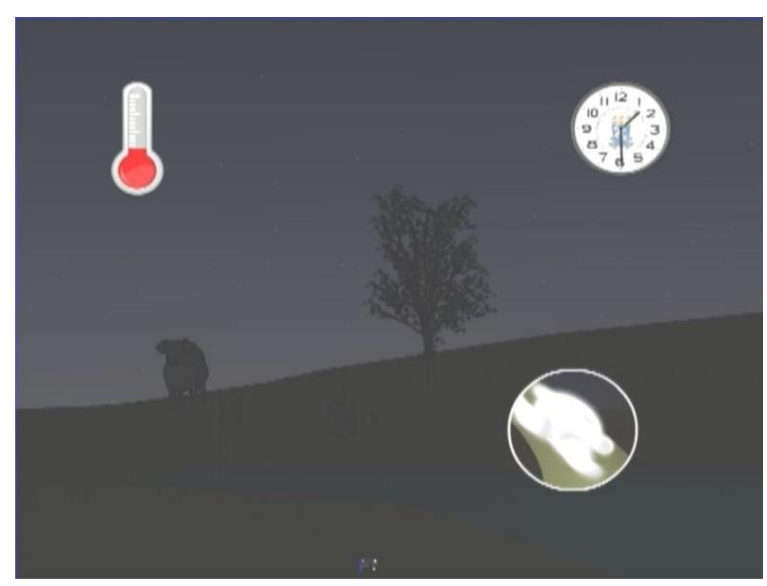

Figura 7: Tela do objeto de aprendizagem no momento da demonstração do processo de formação da geada.

Fonte: dos autores.

Seguindo-se ao processo de aplicação do objeto de aprendizagem, buscou-se avaliar a eficácia do objeto proposto em relação ao aprendizado dos alunos. Procuraram-se perceber a inserção do objeto no ensino dos conteúdos envolvidos, sua utilização e interação com os alunos envolvidos. As figuras 8 e 9 ilustram os resultados obtidos pela avaliação quantitativa.

A partir dos resultados visualizados na figura 9, é possível afirmar que houve resposta positiva com a aplicação do objeto de aprendizagem. Credita-se esse resultado, entre outras razões, ao envolvimento dos alunos com os conteúdos e a eficácia do objeto de aprendizagem. 


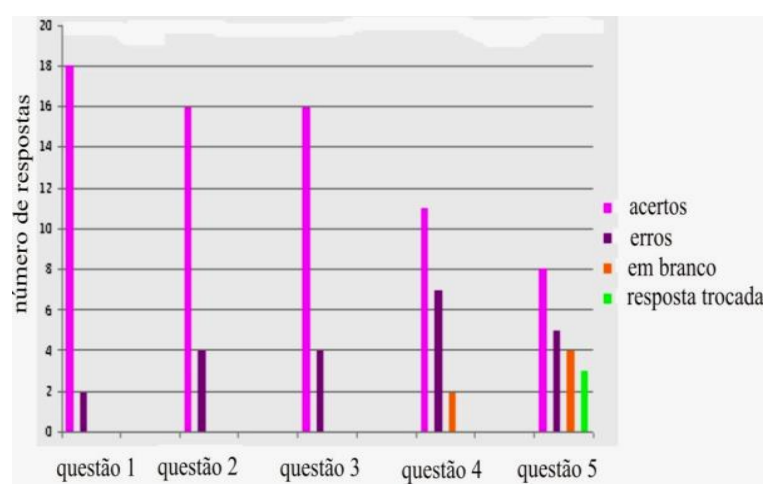

Figura 8: Resultados obtidos pela avaliação quantitativa antes da aplicação do objeto de aprendizagem.

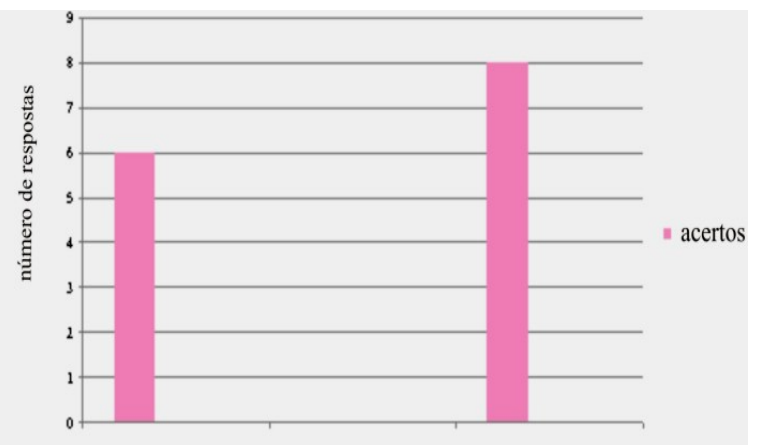

respostas corretas antes da aplicação do objeto respostas corretas após a aplicação do objeto Figura 9: Comparação dos resultados obtidos pela avaliação quantitativa antes e após a aplicação do objeto de aprendizagem.

Ainda, numa última etapa do trabalho, visou avaliar a inserção do objeto de aprendizagem no ensino dos conteúdos escolares e a sua utilização. Nesse sentido, os alunos foram solicitados a responderem sobre a experiência vivenciada. A partir das respostas obtidas, avaliou-se que o objeto mostrou resultado positivo. Teve aceitação unânime demonstrando que recursos alternativos, como a utilização de multimídias digitais alcança grande aceitação. Isso ficou demonstrado pelo grande interesse dos alunos durante a elaboração e aplicação do objeto de aprendizagem, validando-se assim o objeto de aprendizagem.

\section{Conclusão}

Diante dos objetivos propostos e dos resultados obtidos considerou-se eficaz a utilização do objeto de aprendizagem. Também se avalia como positiva a inserção do objeto de aprendizagem para alunos do $6^{\mathrm{o}}$ ano do ensino fundamental. Conclui-se ainda, que a inserção de mídias digitais na forma de objetos de aprendizagem para o estudo do orvalho e da geada constituem um recurso válido no processo de construção aprendizagem, dinamizando de forma interativa a aula. $\mathrm{O}$ objeto de aprendizagem para o estudo do orvalho e da geada proporcionou interesse unânime por parte dos alunos, o que pode ser traduzido como significativo ganho de qualidade para a aula. Conclui se, portanto, que o objeto de aprendizagem foi um recurso válido para auxílio ao professor e no processo de ensinoaprendizagem.

\section{Agradecimentos}

Agradecimentos aos alunos do $6^{0}$ ano, à professora de Geografia e à direção da Escola Municipal de Ensino Fundamental Professora Maria de Lourdes Bandeira Medina. Agradecimentos também ao Programa PROLICEN da Universidade Federal de Santa Maria.

\section{Referências}

Bolfe, S. ; FantinI, V.; Costa, .R. (2007). O ensino da cartografia digital para alunos da $5^{\text {a }}$ série do Ensino Fundamental com o uso do programa GPS Track Maker Free ${ }^{\circledR}$ como recurso didáticopedagógico. In: XXVII ENCONTRO ESTADUAL DE GEOGRAFIA. Anais...Santa Maria: Centro Universitário Franciscano -UNIFRA - AGB. Santa Maria.

Mercado, P. (2002). Novas tecnologias na educação: reflexões sobre a prática. Maceió: EDUFAL.

Niskier, A. (1985). Nova Educação: entre o coração e a máquina. Rio de Janeiro: Bloch Editoras. p.215.

Sosteric, N; Hesemeier, S. (2002) "When is a Learning Object not an Object: a first step towards a theory of learning objects". In: Internacional Review of Research in Open and Distance Learning. Outubro de 2002. Disponível em $<$ http://www.irrodl.org/content/v3.2/soches.html>. Acesso em 10 de fevereiro de 2008.

Tarouco, M.; Fabre, C.; Tamusiunas, F.(2003). Reusabilidade de objetos educacionais. Disponível em $<$ http://www.cinted.ufrgs.br/renote/fev2003/artigo s/marie_reusabilidade.pdf.> Acesso em 20 de julho de 2006. 